\title{
5ANO94-1653C \\ NON-DESTRUCTIVE CHARACTERIZATION OF POROUS SILICON \\ USING X-RAY REFLECTIVITY
}

\author{
E. CHASON, T.R. GUILINGER, M.J. KELLY, T.J. HEADLEY and A.J. HOWARD \\ Sandia National Laboratories, Albuquerque, NM 87185
}

\begin{abstract}
Understanding the evolution of porous silicon (PS) layers at the early stages of growth is important for determining the mechanism of PS film growth and controlling the film properties. We have used X-ray reflectivity (XRR) to determine the evolution of layer thickness and interfacial roughness during the growth of thin PS layers $(<200 \mathrm{~nm})$ prepared by electrochemical anodization. The porous layer grows at a constant rate for films as thin as $15 \mathrm{~nm}$ indicating a very short incubation period during which the surface may be electropolished before the PS structure begins to form. Interface roughness measurements indicate that the top surface of the film remains relatively smooth during growth while the roughness of the PS/silicon interface increases only slightly with film thickness. The XRR results are compared with results obtained from the same films by cross-sectional transmission electron microscopy (XTEM), aomic force microscopy (AFM) and gravimetry.
\end{abstract}

\section{INTRODUCTION}

Porous silicon (PS) films have properties that make them interesting for a variety of technological applications, e.g., as insulating layers in microelectronics [1] and for micromachining applications [2]. The discovery of visible photoluminescence by Canham [3] has generated further interest because of the possibility of creating light emitting devices in Si-based materials. Although there has been significant interest in utilizing PS, there is still not a complete understanding of the porous layer formation process or the resulting microstructure. Part of the reason for this is the difficulty of characterizing the porous structure accurately. Many- of the available techniques are time-consuming and sample preparation can be difficult.

In this paper, we report on the use of X-ray reflectivity (XRR) to study the evolution of the PS film structure. XRR is a sensitive probe of thin film layer thickness and surface and interface roughness. The technique is non-destructive, requires minimal sample preparation and, because $\mathrm{X}$-rays are highly penetrating, it is possible to study buried interfaces. We have concentrated primarily on the evolution of the PS film structure during the very early stages of film formation by measuring a series of samples prepared under identical condition for different anodization times. For comparison of the resolution of XRR with other techniques, some of the samples were also measured using gravimetry, XTEM and AFM.

The porous silicon (PS) was formed by electrochemical anodization of 0.5-1.0 $\Omega-\mathrm{cm}$, boron-doped, (100) p-Si in a dual tank electrochemical cell. Details of the design and testing of this cell are given elsewhere [4]. The PS samples were formed in $5 \mathrm{wt} \% \mathrm{HF}$ at $0.58 \mathrm{~mA} / \mathrm{cm}^{2}$ for times ranging from 5 to $376 \mathrm{~s}$. The total area of PS formed was $45.8 \mathrm{~cm}^{2}$.

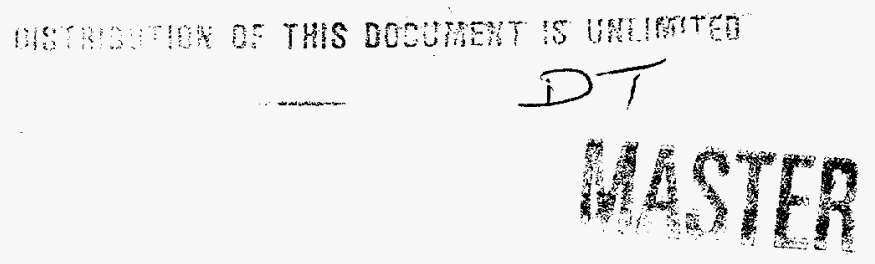




\section{DISCLAIMER}

This report was prepared as an account of work sponsored by an agency of the United States Government. Neither the United States Government nor any agency thereof, nor any of their employees, make any warranty, express or implied, or assumes any legal liability or responsibility for the accuracy, completeness, or usefulness of any information, apparatus, product, or process disclosed, or represents that its use would not infringe privately owned rights. Reference herein to any specific commercial product, process, or service by trade name, trademark, manufacturer, or otherwise does not necessarily constitute or imply its endorsement, recommendation, or favoring by the United States Government or any agency thereof. The views and opinions of authors expressed herein do not necessarily state or reflect those of the United States Government or any agency thereof. 


\title{
DISCLAIMER
}

\begin{abstract}
Portions of this document may be illegible in electronic image products. Images are produced from the best available original document.
\end{abstract}




\section{X-RAY REFLECTIVTTY (XRR)}

$\mathrm{X}$-ray reflectivity is a technique for measuring the structure of thin films. A review of the technique may be found in ref. 5 , so only a brief discussion is given here. The reflectivity is . measured as a function of the scattering vector,

$$
\mathrm{k}=4 \pi / \mathrm{hc} E \sin \theta
$$

where $\mathrm{E}$ is the energy of the $\mathrm{X}$-ray and $2 \theta$ is the scattering angle. In contrast with X-ray diffraction, $\mathrm{X}$-ray reflectivity is performed at small values of $\mathrm{k}$ where the reflectivity may be interpreted as arising from a continuous medium with an index of refraction, $\mathrm{n}$, that depends on the electron density, $\rho_{\mathrm{el}}[6]$. The index of refraction for X-rays in matter is less than 1 so that for sufficiently small incident angles total external reflection occurs.

Above the critical value for total external reflection $\left(k_{c}\right)$, the reflectivity from an ideal interface is given by the Fresnel reflectivity $\left(R_{F}(k)\right)$ with the asymptotic form $\left(k / k_{c}\right)^{-4}$. For an imperfect interface, the reflectivity is given approximately by [5]

$$
R(k)=R_{F}(k)\left|F\left(d \rho_{e} / d z\right)\right|^{2}
$$

where $\mathrm{F}\left(\mathrm{d} \rho_{\mathrm{el}} / \mathrm{dz}\right)$ is the Fourier transform of the electron density gradient in the direction normal to the surface of the film. The relationship between the film structure and eq. (2) is shown schematically in figure 1 . The electron density in the direction normal to the film surface (fig. 1a) is constant where the film composition is uniform and changes at the interfaces between the substrate, the PS film and vacuum. The gradient of the electron density $\left(\mathrm{d} \rho_{\mathrm{e}} / \mathrm{dz}\right)$ has peaks at these interfaces as shown in fig. $1 \mathrm{~b}$. If the interface is smooth, then the peak in $\mathrm{d} \rho_{\mathrm{el}} / \mathrm{dz}$ will be narrow, while if the interface is rough or diffuse, the peak will be broader. The roughness is generally taken to have a Debye-Waller form $\left(\exp \left(-\mathrm{k}^{2} \sigma^{2}\right)\right.$ where $\sigma$ is the interface roughness. The normalized reflectivity from this structure (fig. 1c) is given by the Fourier transform of the density gradient. The oscillations shown in the reflectivity spectra come from interference between scattering from the surface and the buried interface, while the decay in the reflected intensity is determined by the roughness of the interfaces. The reflectivity spectra can therefore be analyzed to obtain the thickness, electron density and interface roughness of the individual layers.

The details of the experimental apparatus are discussed in ref. 7. In the energy dispersive technique used in this work, a broad range of X-ray energies impinge on the sample at a fixed angle and a solid-state Ge detector is used to simultaneously measure the reflectivity at each

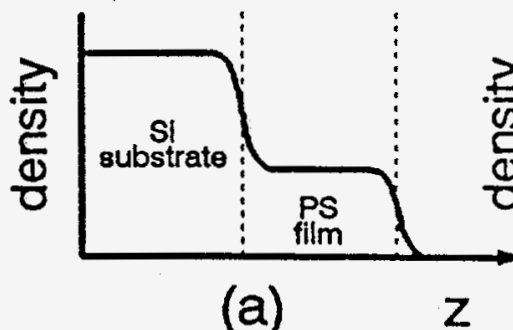

(a)

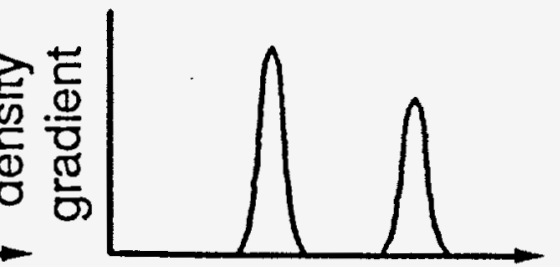

(b)

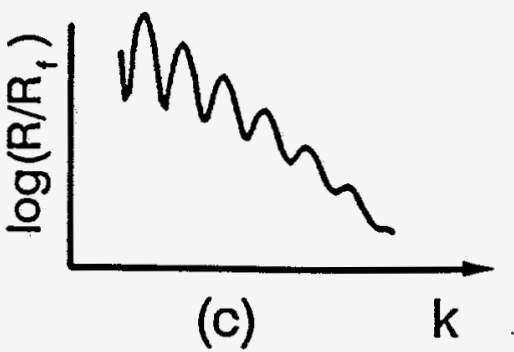

Figure 1. Schematic of relationship between a) electron density of thin film on substrate, b) electron density gradient normal to the surface and $c$ ) normalized $X$-ray reflectivity. $z$ and $k$ are in the direction normal to the surface of the film. 
wavevector $k$. For the actual data analysis, an optical multilayer theory [8] that takes into account multiple scattering is used. Optimum values of parameters corresponding to porosity, PS layer thickness, surface roughness and buried interface roughness were obtained using a non-linear least-squares fitting routine. It is important to note that XRR only measures variations in the electron density normal to the film surface and does not probe the interface structure in the plane of the film. The total area of the sample illuminated by the X-ray beam is approximately $0.5 \times 0.5$ $\mathrm{cm}^{2}$; the measured roughness corresponds to integrating over a region determined by the lateral coherence length of the X-rays which is on the order of $1 \mu \mathrm{m}$.

XRR spectra are shown in figure 2 from PS samples prepared for anodization periods of $5,15,30,60$ and $140 \mathrm{~s}$ as indicated in the figure. The increase in the frequency of the intensity oscillations with the anodization time corresponds to increasing layer thickness. The oscillations decay after only a few periods indicating substantial roughness at one or both of the interfaces. The solid lines represent the calculation of the reflectivity from the optical multilayer theory. For the sample that was processed for $5 \mathrm{~s}$, the structure could be adequately described by a model that contained only a rough surface and no PS layer. For the other samples, a model structure containing a single PS layer on the Si substrate was used.

The dependence of the thickness of the PS layer on the anodization time is shown in figure 3. The error in the XRR determination of layer thickness is estimated to be $\pm 5 \%$. The evolution of the roughness with anodization time is shown in figure $4 \mathrm{a}$ for the roughness from the surface and in figure $4 \mathrm{~b}$ for the buried PS/substrate interface. The error on the surface roughness is estimated to be $\pm 0.2 \mathrm{~nm}$ while the error on the buried interface roughness is $\pm 0.5 \mathrm{~nm}$. The solid lines in figures 3 and 4 are guides to the eye.

In the following section, we present a comparison between results obtained using XRR and other thin film analytical techniques. The implications of these results for understanding the growth process will be discussed in the final section.

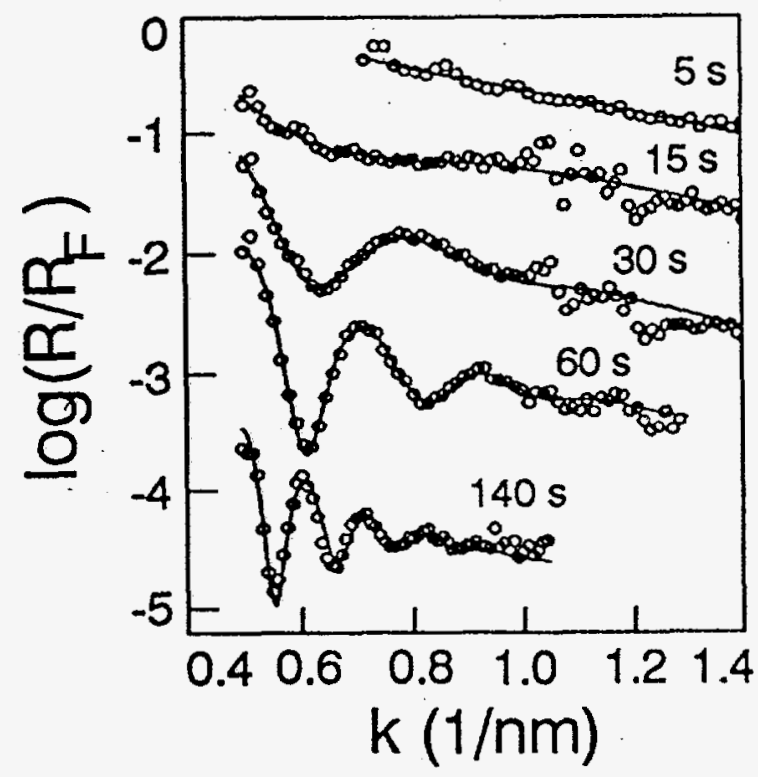

Figure 2. X-ray reflectivity spectra from PS films. Open circles are experimental data; solid lines are calculations from optical multilayer theory. Anodization times are indicated on the figure.

\section{COMPARISON OF XRR AND OTHER EXPERIMENTAL TECHNIQUES}

In order to compare the sensitivity of the XRR technique to other thin film analytical methods, a series of samples were prepared with different anodization times and analyzed using XRR, XTEM, AFM and gravimetry. An XTEM micrograph for a sample that was anodized for 


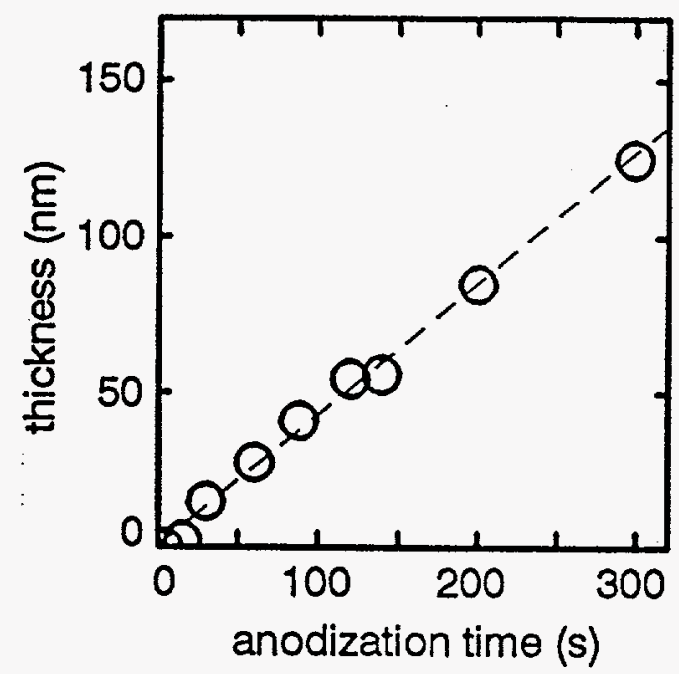

Figure 3. PS layer thickness vs. anodization time as determined by XRR.

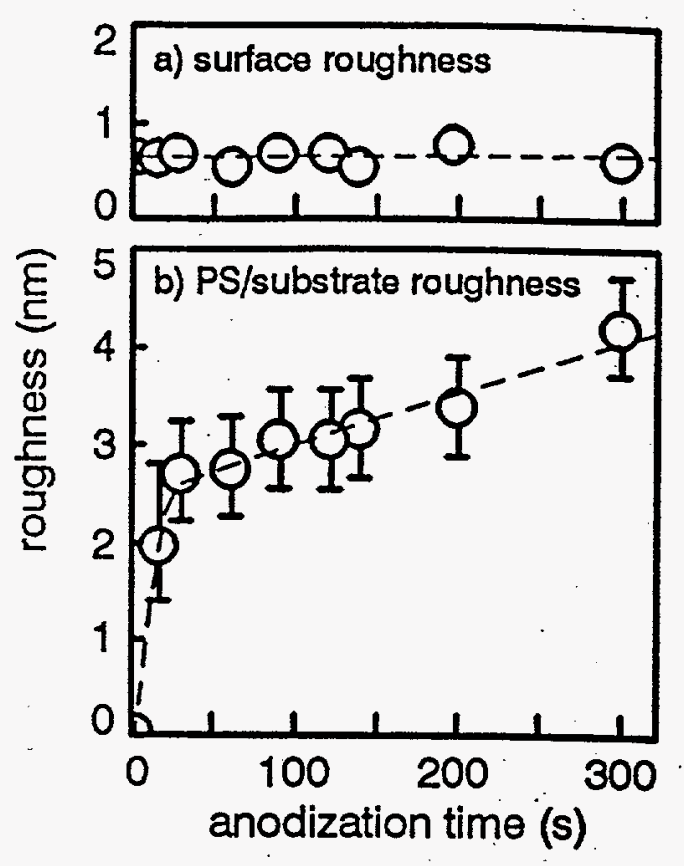

Figure 4. Development of a) surface roughness and b) PS/substrate interfacial roughness with anodization time as determined by XRR.

$141 \mathrm{~s}$ is shown in figure 5. The XTEM measurements were digitized and the average PS layer thickness and RMS roughness at the surface and buried interface was calculated. The lateral range covered by the micrograph was approximately $500 \mathrm{~nm}$, which is comparable to the lateral coherence length of the XRR method. The micrograph indicates that the bottom interface is significantly rougher than the top surface. The image also shows no gross non-uniformities in the layer which is consistent with the good agreement of the XRR spectra with a single layer model.

Gravimetric measurements of layer thickness and porosity are obtained by measuring the change in the sample weight before and after PS layer formation and after stripping off the PS layer using $\mathrm{NaOH}$. The porosity measurements (not shown) indicate that the porosity of the PS layer does not increase significantly with increasing layer thickness. The porosity of these films was on the order of $70-80 \%$. The XRR results are consistent with the porosity of the films being independent of the layer thickness, but because of the large interface roughness the errors on the XRR determination of the porosity are large.

AFM measurements of the surface roughness were obtained from analysis of a $1 \mu \mathrm{m} \times 1$ $\mu \mathrm{m}$ region, comparable to the coherence length of the $X$-rays. The buried interface roughness was obtained by imaging the surface after stripping off the PS layer with $7 \mathrm{M} \mathrm{NaOH}$.

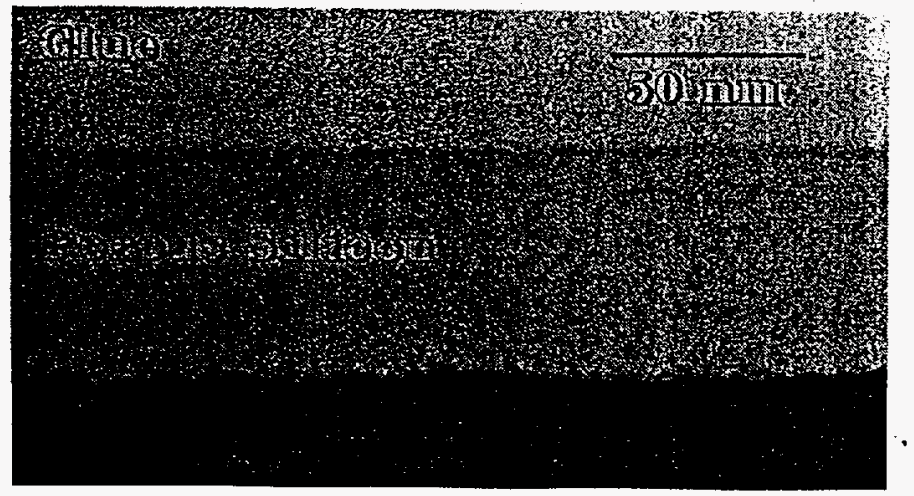

Figure 5. Cross-sectional TEM micrograph of PS film after $141 \mathrm{~s}$ anodization time. 
The results for the layer thickness determination are shown in figure 6 . The thicknesses calculated by gravimetry are consistently higher than for the XRR and XTEM. At small values of the layer thickness, the agreement of the gravimetry results becomes worse. For these very small layer thicknesses, the measurement is presumably dominated by the resolution of the weight change measurement. The agreement between the XRR and XTEM determinations of the sample thickness is very good. The main drawback to the XTEM analysis is the difficulty of preparing cross-sectional samples for analysis. Because XTEM samples a very small portion of the sample, local variations in the thickness can have a significant effect on the errors associated with the measurement.

A comparison of the measured surface and interface roughness is shown in figure 7 for the surface (figure 7a) and the PS/substrate interface (figure 7b). All of the techniques indicate that the bottom interface is significantly rougher than the surface and are in approximate agreement on the magnitude of the interfacial $(2-5 \mathrm{~nm})$ and surface $(0.5 \mathrm{~nm})$ roughness. Although XTEM suggests a stronger dependence of the bottom surface roughness on anodization time than the XRR, the small area of the sample used for XTEM measurements makes it difficult to determine what error should be associated with this number. The AFM value of the bottom surface roughness also may be supressed due to smoothing of the interface during the PS removal with $\mathrm{NaOH}$.

\section{EVOLUTION OF THIN FILM STRUCTURE}

The results of the XRR measurement of PS layer thickness (figure 3) indicate that the layer formation rate is linear with time even for very thin layers. Significant deviation from linearity occurs only for the results obtained at the shortest anodization times: $5 \mathrm{~s}$, where no PS layer is observed, and $15 \mathrm{~s}$ where the measured thickness falls below the linear value extrapolated from the longer times. These results suggest that the growth is linear except when the layer thickness is comparable to the roughness of the bottom interface.

The evolution of the surface roughness with anodization time (figure 4) indicates that once the initial pore formation is completed, the top surface does not continue to etch significantly.

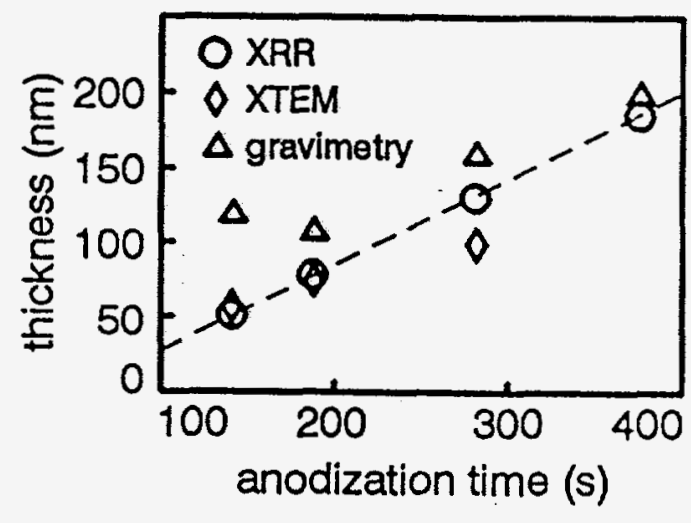

Figure 6. Comparison of PS film thickness determined by XRR, XTEM and gravimetry.

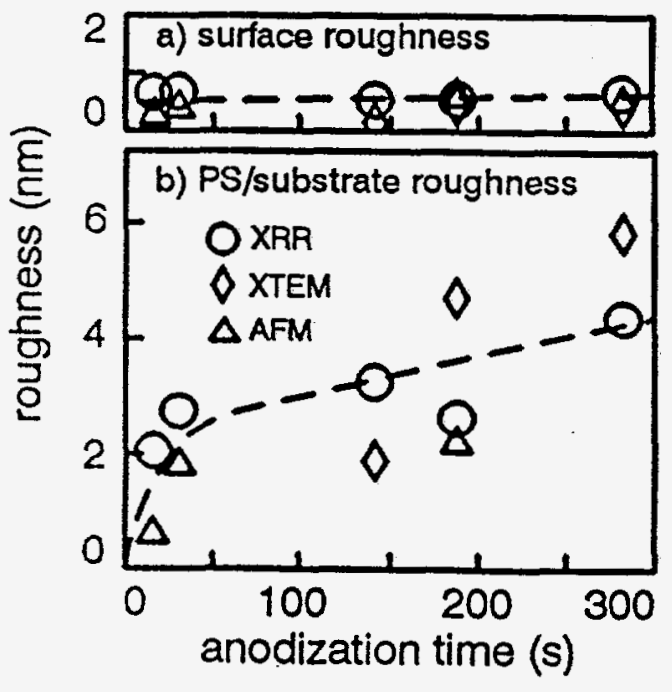

Figure 7. Comparison of a) surface roughness and $b$ ) PS/substrate interfacial roughness determined by XRR, XTEM and AFM. 
The surface reaches a roughness of between $0.5-0.7 \mathrm{~nm}$ after only $5 \mathrm{~s}$, and this roughness does not increase over the measured range. This result is consistent with the gravimetric result that indicates that the porosity does not change after the initial porous layer is formed. If the porosity were changing significantly, it would most likely lead to an increase in the surface roughness as well. Etching of the Si by HF does not appear to contribute to a significant increase in the surface roughness. At an estimated etch rate of $0.0005 \mathrm{~nm} / \mathrm{s}$ [9], only $0.15 \mathrm{~nm}$ of Si is removed after the maximum etch time of $300 \mathrm{~s}$ which is significantly less than the surface roughness of $0.6 \mathrm{~nm}$.

The fact that the roughness of the buried interface does not increase significantly suggests that the PS forming reaction is self-limiting, i.e., that the pores interact to keep the growth front at the same level. This is consistent with previous work performed on the effect of PS formation on initially rough substrates in which it was found that the porous layer formation resulted in a buried interface that was smoother than the intial surface roughness [10].

These results are consistent with a model that suggests that for very early times, the porous layer starts to form by initially roughening the top surface. This porous layer is formed very shortly after the etching process begins since we do not observe a long incubation period of electropolishing before the PS layer formation begins. The $\mathrm{Si}$ appears to be significantly etched only in the vicinity of the pore tips since the top surface does not continue to roughen after the PS layer is formed. The active region of pore formation has a width of approximately $3 \mathrm{~nm}$ which it maintains as it continues to burrow into the Si substrate.

\section{CONCLUSION}

We have used X-ray reflectivity to study the evolution of PS layer thickness, surface roughness and PS/substrate interface roughness. The results obtained with XRR are in good agreement with results obtained by gravimetry, XTEM and AFM. The growth kinetics indicate that the rate of PS formation is remarkably linear beyond the point where the layer thickness is comparable to the steady-state roughness of the PS/substrate interface. The surface and buried interface roughness remain constant during the PS layer growth with the buried interface rougher than the surface by a factor of 5 . This work was performed at Sandia National Laboratories supported by the U. S. Department of Energy under contract DE-ACO4-94AL85000.

\section{REFERENCES}

1. S.S. Tsao, IEEE Circuits Devices CD-3, 3 (1987).

2. P. Steiner, A. Richter and W. Lang, J. Micromech. Microeng. 3, 32 (1993).

3. L.T. Canham, Appl. Phys. Lett. 57, 1046 (1990).

4. T.R. Guilinger, M.J. Kelly and J.O. Stevenson, Proc. of the First Int'l. Symposium on Electrochemical Microfabrication, edited by M. Datta, K. Sheppard and D. Snyder, The Electrochemical Society, vol. 92-3, 1992.

5. J. Als-Nielsen in Structure and Dynamics of Surfaces, edited by W. Schommers and P. von Blanckenhagen (Springer, Berlin, 1986), Chap. 5, p. 181.

6. J.H. Underwood and T.W. Barbee, Appl. Opt. 20, 3027 (1981).

7. E. Chason, T.M. Mayer, A. Payne and D.T. Wu, Appl. Phys. Lett. 60, 2353 (1992).; E. Chason and D.T. Warwick, Mat. Res. Symp. Proc. Vol 208, 351 (1991).

8. L.G. Parratt, Phys. Rev. 95, 359 (1954).

9. S.M. Hu and D.R. Kerr, J. Electrochem. Soc. 114, 414 (1967).

10. unpublished result. 